\title{
Article
}

\section{Skeletal Muscle Volume Is an Independent Predictor of Survival after Sorafenib Treatment Failure for Hepatocellular Carcinoma}

\author{
Issei Saeki ${ }^{1}{ }^{\circledR}$, Takahiro Yamasaki ${ }^{2, *} \mathbb{D}$, Yurika Yamauchi ${ }^{1}$, Taro Takami ${ }^{1}{ }^{\circledR},{ }$ Tomokazu Kawaoka ${ }^{3}$, \\ Shinsuke Uchikawa ${ }^{3}{ }^{\circledR}$, Akira Hiramatsu ${ }^{3}$, Hiroshi Aikata ${ }^{3}$, Reo Kawano ${ }^{4}{ }^{\circ}$, Kazufumi Kobayashi ${ }^{5,6}$, \\ Takayuki Kondo ${ }^{5}$, Sadahisa Ogasawara ${ }^{5,6}{ }^{\circledR}$, Tetsuhiro Chiba ${ }^{5}$, Kazuaki Chayama ${ }^{3}$, Naoya Kato ${ }^{5}$ \\ and Isao Sakaida ${ }^{1}$
}

Citation: Saeki, I.; Yamasaki, T.; Yamauchi, Y.; Takami, T.; Kawaoka, T.; Uchikawa, S.; Hiramatsu, A.; Aikata, H.; Kawano, R.; Kobayashi, K.; et al. Skeletal Muscle Volume Is an Independent Predictor of Survival after Sorafenib Treatment Failure for Hepatocellular Carcinoma. Cancers 2021, 13, 2247. https://doi.org/ 10.3390/cancers13092247

Academic Editors: Alessandro Vitale, Quirino Lai and David A. Geller

Received: 24 March 2021

Accepted: 28 April 2021

Published: 7 May 2021

Publisher's Note: MDPI stays neutral with regard to jurisdictional claims in published maps and institutional affiliations.

Copyright: (c) 2021 by the authors. Licensee MDPI, Basel, Switzerland. This article is an open access article distributed under the terms and conditions of the Creative Commons Attribution (CC BY) license (https:// creativecommons.org/licenses/by/ $4.0 /)$.
1 Department of Gastroenterology and Hepatology, Yamaguchi University Graduate School of Medicine, Yamaguchi 755-8505, Japan; issaeki@yamaguchi-u.ac.jp (I.S.); g030ub@yamaguchi-u.ac.jp (Y.Y.); t-takami@yamaguchi-u.ac.jp (T.T.); sakaida@yamaguchi-u.ac.jp (I.S.)

2 Department of Oncology and Laboratory, Yamaguchi University Graduate School of Medicine, Yamaguchi 755-8505, Japan

3 Department of Gastroenterology and Metabolism, Graduate School of Biomedical and Health Sciences, Hiroshima University, Hiroshima 734-8551, Japan; kawaokatomo@hiroshima-u.ac.jp (T.K.); shinuchi@hiroshima-u.ac.jp (S.U.); akirah@hiroshima-u.ac.jp (A.H.); aikata@hiroshima-u.ac.jp (H.A.); chayama@hiroshima-u.ac.jp (K.C.)

4 Clinical Research Center in Hiroshima, Hiroshima University Hospital, Hiroshima 734-8551, Japan; rkawano@hiroshima-u.ac.jp

5 Department of Gastroenterology, Graduate School of Medicine, Chiba University, Chiba 260-8667, Japan; kobayashi-kazufumi@chiba-u.jp (K.K.); takakon@chiba-u.jp (T.K.); ogasawaras@chiba-u.jp (S.O.); chibat@chiba-u.jp (T.C.); kato.naoya@chiba-u.jp (N.K.)

6 Translational Research and Development Center, Chiba University Hospital, Chiba 260-8667, Japan

* Correspondence: t.yama@yamaguchi-u.ac.jp; Tel.: +81-836-22-2337

Simple Summary: Skeletal muscle volume has been reported as a prognostic factor for patients with hepatocellular carcinoma receiving sorafenib. In this study, we show that skeletal muscle volume is not only a predictor of overall survival but also of post-progression survival, which represents survival time following confirmation of progressive disease. We may be able to prolong survival by upregulating skeletal muscle volume, especially in hepatocellular carcinoma patients with skeletal muscle depletion.

Abstract: Few studies exist on the relationship between post-progression survival (PPS) and skeletal muscle volume in hepatocellular carcinoma (HCC) patients receiving sorafenib. This study aimed to analyze the effects of muscle volume on clinical outcomes. We retrospectively enrolled 356 HCC patients. Various clinical parameters, including skeletal muscle index, were analyzed as predictors of overall survival (OS), progression-free survival (PFS), and PPS. Patients with high muscle volume showed longer survival or PPS than those with low muscle volume (median survival time: $12.8 \mathrm{vs}$. 9.5 months, $p=0.005$; median PPS: 8.2 vs. 6.3 months, $p=0.015)$; however, no differences in PFS were found. Multivariate analysis indicated that muscle volume was an independent predictor of PPS and OS. Skeletal muscle volume was a PPS predictor in HCC patients receiving sorafenib. Therefore, survival can be prolonged by the upregulation of skeletal muscle volume, especially in HCC patients with skeletal muscle depletion.

Keywords: hepatocellular carcinoma; muscle depletion; post-progression survival; sorafenib

\section{Introduction}

Numerous molecular-targeted agents (MTAs) and immune checkpoint inhibitors (ICIs) have been introduced for treating hepatocellular carcinoma (HCC). Sorafenib was approved in 2007 as a first-line drug for the systemic treatment of advanced HCC [1] and has played 
a significant role in the development of clinical trials. However, compared to treatment with sorafenib, treatment with a combination of atezolizumab and bevacizumab led to a greater improvement in overall survival (OS) and progression-free survival (PFS) [2]; thus, this combination therapy can be considered as the first-line therapy for advanced HCC. Although OS is commonly used as the primary outcome in clinical trials for HCC [1-6], post-progression survival (PPS) is an important factor for prolonging OS. Moreover, the RESORCE study and a sub-analysis of the REFLECT study demonstrated that sequential therapy improved survival in patients who were refractory to the first-line therapy $[3,7]$. Furthermore, PPS was highly correlated with OS in HCC patients receiving sorafenib [8]. Several predictive biomarkers of OS are reported in HCC patients receiving sorafenib, but there are no useful PPS predictors in such patients [9]. It is possible to evaluate these in HCC patients receiving sorafenib who have long follow-up periods.

Sarcopenia was first defined in 1989 as age-related skeletal muscle depletion [10,11]; studies have shown that it is caused by various cancers, including HCC [12-14]. Sarcopenia diagnosis criteria include impaired physical performance and loss of muscle volume and strength in the aged population $[15,16]$. However, skeletal muscle depletion has been the most commonly used definition for sarcopenia $[13,14]$ and has been reported as a poor prognostic factor in HCC patients receiving sorafenib [17-19]. To the best of our knowledge, there are no studies on the relationship between PPS and skeletal muscle volume before sorafenib treatment for HCC.

In this multicenter study, we retrospectively investigated the effects of skeletal muscle volume on clinical outcomes such as OS, PFS, and PPS in HCC patients receiving sorafenib.

\section{Materials and Methods}

\subsection{Study Population}

Patients who received treatment with sorafenib at three hospitals in Japan (the Chiba University Hospital, Hiroshima University Hospital, and Yamaguchi University Hospital) between April 2009 and July 2016 were included in this study. HCC diagnosis was based on radiological or pathological findings and expression of tumor markers (alphafetoprotein and des-gamma-carboxy prothrombin). The inclusion criteria were as follows: (i) unresectable HCC confirmed by a pathological or imaging diagnosis; (ii) stage $\mathrm{B}$ or $\mathrm{C}$ tumor according to the Barcelona Clinic Liver Cancer (BCLC) staging system [20]; and (iii) computed tomography (CT) data available for up to the third lumbar vertebra before sorafenib treatment. Patients with incomplete data were excluded. As shown in Figure S1, a total of 546 patients received sorafenib during the study period. Among these, clinical data and information on skeletal muscle volume were lacking in 152 patients, while 38 did not undergo radiological imaging during treatment. Thus, OS or PFS were evaluated only in 356 patients. Of these, 36 patients did not develop radiological tumor progression until the last follow-up date; hence, PPS was analyzed in 320 patients. This study was approved by the Institutional Review Board of the Yamaguchi University Hospital (H30-042) and those of the two other institutions and was performed in accordance with the ethical principles of the 1975 Declaration of Helsinki. Informed consent was not obtained due to the retrospective study design.

\subsection{Treatment}

In principle, the daily starting dose of sorafenib was $800 \mathrm{mg}$, but it was reduced depending on the patient's liver function and/or general status. Sorafenib treatment was continued until the patient experienced tumor progression, unacceptable adverse events, or death. Post-progression treatments, such as transcatheter arterial chemoembolization (TACE), hepatic resection, hepatic arterial infusion chemotherapy (HAIC), and other systemic therapies were performed as per the patient's liver function, tumor burden, and performance status, as well as at the discretion of each hospital. 


\subsection{Assessment of Skeletal Muscle Volume}

All patients underwent CT within 1 month before sorafenib treatment. Radiological data were collected as Digital Imaging and Communication in Medicine data at the Yamaguchi University Hospital. At this hospital, skeletal muscle volume was measured at the level of the third lumbar vertebra using an AZE 3D workstation (AZE Virtual Place Raijin; AZE Ltd., Tokyo, Japan) and was categorized on the basis of a CT radiodensity value of -29 to $+150 \mathrm{HU}$ [21]. Muscle area was standardized as the square of height, and skeletal muscle index (SMI) was calculated by dividing the skeletal muscle mass by the square of height. Skeletal muscle volume was classified into high (H-MV) and low (L-MV) muscle volume using the median SMI in males or females as the threshold.

\subsection{Evaluation of Treatment Response}

Treatment response was evaluated according to the Response Evaluation Criteria in Solid Tumors [22] and based on dynamic CT or dynamic magnetic resonance imaging every $2-3$ months. The best response was used for response evaluation.

\subsection{Statistical Analyses}

Continuous variables are expressed as medians and interquartile ranges (IQRs). Between-group comparisons were performed using the chi-squared test or the Fisher's exact test. OS, PFS, and PPS were calculated using the Kaplan-Meier method. OS was defined as the interval from the start of sorafenib treatment to death, last visit, or last follow-up. PFS was defined as the interval from the start of sorafenib treatment to the first radiological confirmation of progressive disease (PD) or death. PPS was defined as the interval from PD confirmation to death, last visit, or last follow-up. The follow-up period ended on 31 March 2018. A total of 273 patients died during the study period, and Spearman's test was used to estimate the correlation between OS and PFS/PPS in them.

To define the OS, PFS, and PPS predictive factors, we assessed the following baseline parameters before sorafenib treatment: age ( $<70$ years or $\geq 70$ years), sex (male/female), body mass index (BMI) $\left(\geq 22 \mathrm{~kg} / \mathrm{m}^{2}\right.$ or $\left.<22 \mathrm{~kg} / \mathrm{m}^{2}\right)$, Eastern Cooperative Oncology Group performance status (ECOG-PS) $(0,1$, or 2), Child-Pugh class (A/B), tumor number $(<8$ or $\geq 8$ ), tumor size ( $<35 \mathrm{~mm}$ or $\geq 35 \mathrm{~mm}$ ), macrovascular invasion (MVI) (absence/presence), extrahepatic spread (EHS) (absence/presence), and skeletal muscle volume (high/low). Median values were used as the threshold for age, tumor size, and tumor number. The threshold for BMI, which is considered standard due to having the lowest morbidity [23], was used. Furthermore, to analyze the prognostic parameters of OS in the 320 patients who were refractory to sorafenib treatment, we included two factors in addition to the aforementioned parameters: disease control with sorafenib (yes/no) and post-sorafenib therapy (yes/no). Univariate and multivariate analyses of the predictive factors were performed using the Cox proportional hazard model and logistic regression analysis; the results are presented as hazard ratios (HRs) or odds ratios (ORs) with $95 \%$ confidence intervals (CIs). All statistical analyses were performed using JMP Pro 15 software (SAS Institute Inc., Cary, NC, USA). Statistical significance was defined at a $p$-value $<0.05$.

\section{Results}

\subsection{Patient Characteristics}

The patients' profiles are summarized in Table 1. A total of 310 and 46 patients had a Child-Pugh class of A and B, respectively. In terms of BCLC staging, 78 and 278 patients were classified as having stage $B$ and $C$ tumors, respectively. The median SMI was $45.3 \mathrm{~cm}^{2} / \mathrm{m}^{2}$ in males and $38.3 \mathrm{~cm}^{2} / \mathrm{m}^{2}$ in females. Therefore, L-MV was defined as an SMI of $<45 \mathrm{~cm}^{2} / \mathrm{m}^{2}$ in males and $<38 \mathrm{~cm}^{2} / \mathrm{m}^{2}$ in females. 
Table 1. Patient characteristics.

\begin{tabular}{cc}
\hline Factors & Total $(\boldsymbol{n}=\mathbf{3 5 6})$ \\
Age & $69.5(63.0-75.0)$ \\
Sex $($ male/female) & $287 / 69$ \\
Etiology $(\mathrm{HCV} / \mathrm{HBV} / \mathrm{HBV}+\mathrm{HCV} / \mathrm{NBNC})$ & $175 / 80 / 2 / 99$ \\
Body mass index $\left[\mathrm{kg} / \mathrm{m}^{2}\right]$ & $22.9(20.8-24.9)$ \\
ECOG-PS $(0 / 1 / 2 / 3)$ & $314 / 37 / 3 / 2$ \\
Child-Pugh class $(\mathrm{A} / \mathrm{B})$ & $310 / 46$ \\
Barcelona Clinic Liver Cancer stage (B/C) & $78 / 278$ \\
Tumor number & $8(2-8)$ \\
Tumor size [mm] & $35.0(18.3-65.0)$ \\
Macrovascular invasion $(-/+)$ & $258 / 98$ \\
Extrahepatic spread $(-/+)$ & $167 / 189$ \\
Response (CR/PR/SD/PD) & $0 / 16 / 197 / 143$ \\
male & $45.3(41.2-50.4)$ \\
female & $38.3(34.0-42.9)$ \\
Muscle volume (high/low) & $181 / 175$ \\
\hline
\end{tabular}
Group performance status; CR, domplete response; PR, partial response; $\mathrm{SD}$, stable disease; PD, progressive disease.

\subsection{Treatment Response and Its Predictors}

A total of $16(4.5 \%), 197(55.3 \%)$, and $143(40.2 \%)$ patients showed partial response (PR), stable disease (SD), and PD, respectively. The objective response rate (ORR) and disease control rate (DCR) were $4.5 \%$ and $59.8 \%$, respectively. Univariate and multivariate analyses demonstrated that absence of MVI contributed to disease control after treatment with sorafenib (OR: 1.750, 95\% CI: 1.028-2.978, $p=0.039$ ), but skeletal muscle volume was not a significant predictor of disease control after treatment with sorafenib (Table S1).

\subsection{OS, PPS, and PFS According to Skeletal Muscle Volume}

The median survival time (MST), median PFS, and median PPS were 11.3, 3.2, and 7.2 months, respectively (Figures 1a, 2a and 3a). Patients with H-MV showed significantly longer survival or PPS than those with L-MV (MST: 12.8 vs. 9.5 months, $p=0.005$, Figure 1b; median PPS: 8.2 vs. 6.3 months, $p=0.015$, Figure 3b). However, there was no significant difference in PFS between patients with H-MV and those with L-MV (median PFS: 3.5 vs. 3.0 months, $p=0.295$, Figure $2 b$ ). We compared the H-MV and L-MV characteristics between patients followed for OS/PFS $(n=356)$ and those followed for PPS $(n=320)$ analysis. There were no significant differences in the factors considered, except for BMI, tumor number, and MVI (Tables S2 and S3).

\subsection{Correlation between $O S$ and PFS/PPS}

We analyzed the correlation between OS and PFS/PPS in 273 patients who died during the study period. PPS was strongly correlated with OS $(\mathrm{R}=0.908, p<0.001)$, while PFS was moderately correlated with OS $(\mathrm{R}=0.642, p<0.001)$ (Figure $\mathrm{S} 2)$.

\subsection{Predictors of PFS}

On multivariate analysis, tumor number $<8$ (HR: $0.611,95 \%$ CI: $0.482-0.770$, $p<0.001$ ) and the absence of EHS (HR: $0.733,95 \%$ CI: 0.582-0.924, $p=0.008$ ) were significant predictors of PFS, whereas skeletal muscle volume was not a significant predictor of PFS $(p=0.255)$ (Table S4).

\subsection{Predictors of PPS or OS}

On multivariate analysis, six factors-male sex (HR: 0.672, 95\% CI: 0.494-0.914, $p=0.011$ ), Child-Pugh class A (HR: 0.612, 95\% CI: 0.421-0.890, $p=0.010$ ), tumor number $<8$ (HR: $0.656,95 \%$ CI: 0.509-0.844, $p=0.001$ ), absence of MVI (HR: 0.720, 95\% CI: $0.528-0.982, p=0.038$ ), absence of EHS (HR: $0.674,95 \%$ CI: $0.521-0.872, p=0.003$ ), and H-MV 
(HR: $0.698,95 \%$ CI: $0.509-0.959, p=0.027$ - —were found to be significant predictors of PPS (Table 2).

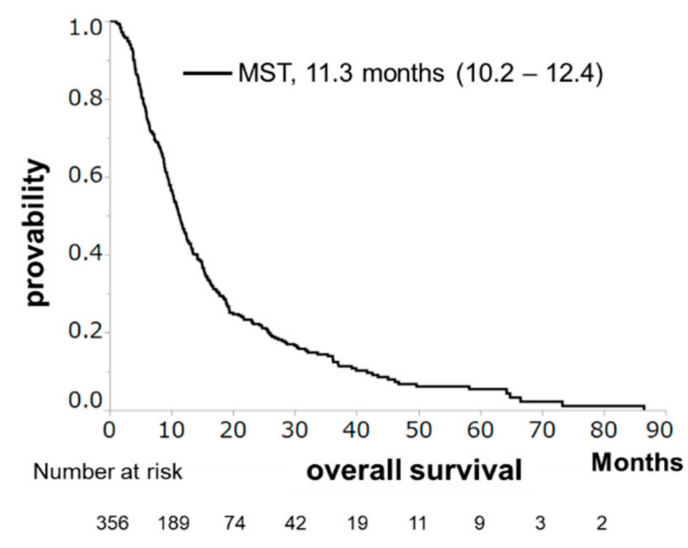

(a)

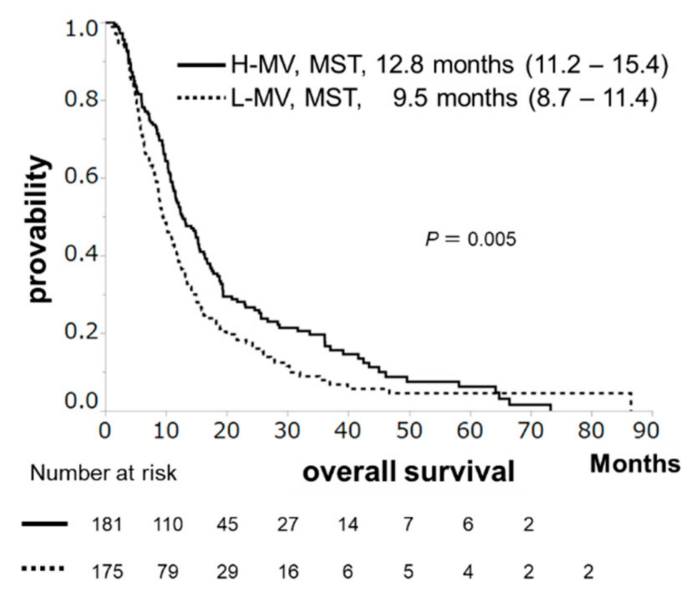

(b)

Figure 1. Overall survival (OS) in hepatocellular carcinoma patients receiving sorafenib. (a) The OS rates at 1, 2, and 3 years are $46.5 \%, 22.2 \%$, and $13.9 \%$, respectively, with a median survival time (MST) of 11.3 months. (b) OS according to skeletal muscle volume. Patients with high muscle volume (H-MV) showed significantly longer survival than those with low muscle volume (L-MV) (MST: 12.8 vs. 9.5 months, $p=0.005$ ).

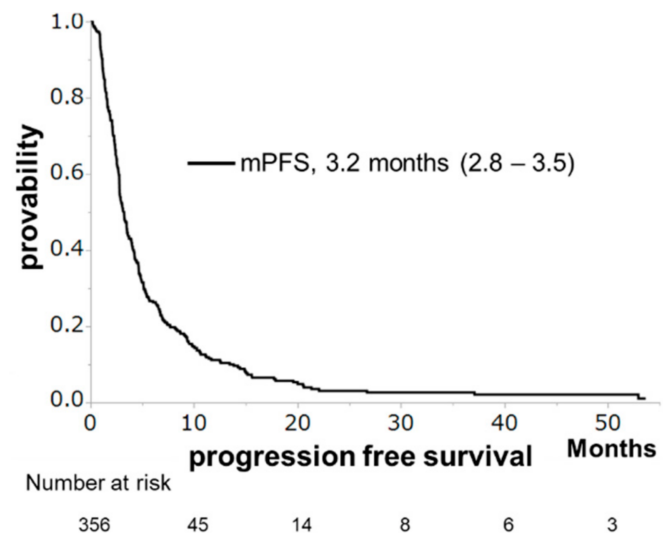

(a)

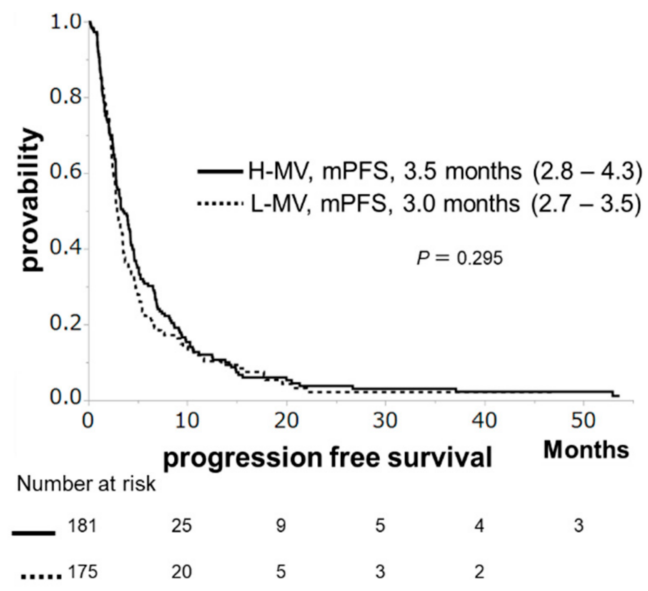

(b)

Figure 2. Progression-free survival (PPS) in hepatocellular carcinoma patients receiving sorafenib. (a) The PFS rates at 1 and 2 years are $11.2 \%$ and $3.1 \%$, respectively, with a median PFS of 3.2 months. (b) PFS according to skeletal muscle volume. No significant difference in PFS can be observed between patients with high muscle volume (H-MV) and those with low muscle volume (L-MV) (median PFS: 3.5 vs. 3.0 months, $p=0.295$ ).

Furthermore, as shown in Table 3, multivariate analysis indicated that these factors were also significant predictors of OS: male sex (HR: 0.661, 95\% CI: 0.491-0.889, $p=0.006$ ), Child-Pugh class A (HR: 0.701, 95\% CI: 0.496-0.992, $p=0.045$ ), tumor number $<8$ (HR: 0.591, 95\% CI: 0.465-0.751, $p<0.001$ ), absence of MVI (HR: 0.678, 95\% CI: 0.506-0.907, $p=0.009$ ), absence of EHS (HR: 0.600, 95\% CI: 0.470-0.766, $p<0.001$ ), and H-MV (HR: 0.666, $95 \%$ CI: 0.501-0.886, $p=0.005)$. 


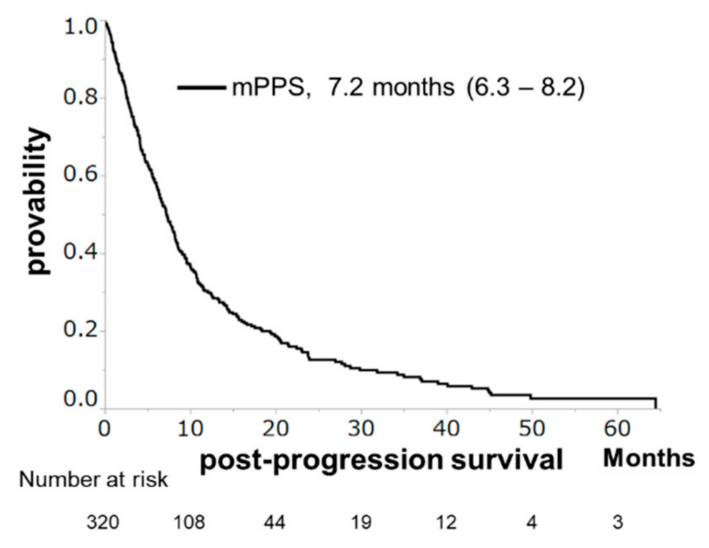

(a)

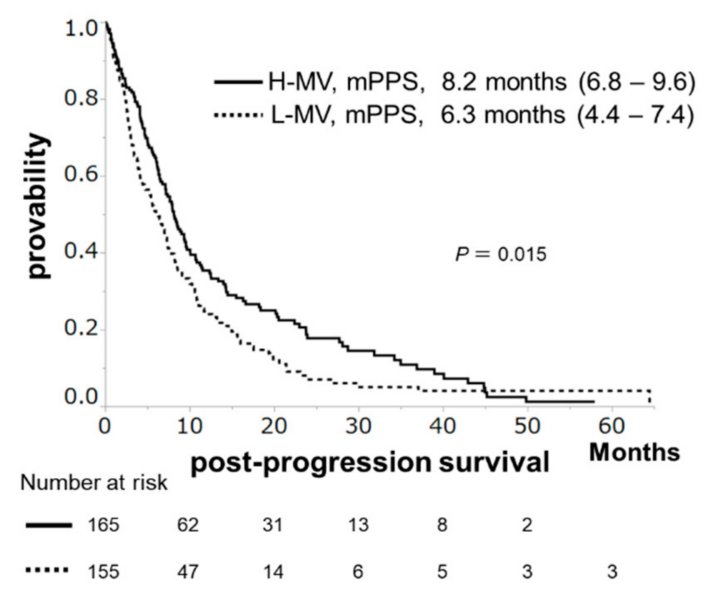

(b)

Figure 3. Post-progression survival (PPS) in hepatocellular carcinoma patients receiving sorafenib. (a) The PPS rates at 1 and 2 years are $30.3 \%$ and $12.6 \%$, respectively, with a median PPS of 7.2 months. (b) PPS according to skeletal muscle volume. Patients with high muscle volume (H-MV) showed significantly longer PPS than those with low muscle volume (L-MV) (median PPS, 8.2 vs. 6.3 months, $p=0.015$ ).

Table 2. Univariate and multivariate analyses for predictors of post-progression survival $(n=320)$.

\begin{tabular}{|c|c|c|c|c|c|c|}
\hline \multirow{2}{*}{ Factors } & \multicolumn{3}{|c|}{ Univariate Analysis } & \multicolumn{3}{|c|}{ Multivariate Analysis } \\
\hline & HR & $95 \% \mathrm{CI}$ & $p$ Value & HR & $95 \% \mathrm{CI}$ & $p$ Value \\
\hline Age $(<70 / \geq 70)$ & 1.135 & $0.893-1.442$ & 0.300 & 1.082 & $0.844-1.386$ & 0.534 \\
\hline Sex (male/female) & 0.742 & $0.549-1.003$ & 0.052 & 0.672 & $0.494-0.914$ & 0.011 \\
\hline Body mass index $\left[\mathrm{kg} / \mathrm{m}^{2}\right](\geq 22 /<22)$ & 0.918 & $0.720-1.170$ & 0.488 & 1.155 & $0.849-1.570$ & 0.360 \\
\hline ECOG-PS $(-1 / 2-)$ & 0.607 & $0.250-1.476$ & 0.271 & 0.633 & $0.257-1.555$ & 0.318 \\
\hline Child-Pugh class (A/B) & 0.617 & $0.432-0.880$ & 0.008 & 0.612 & $0.421-0.890$ & 0.010 \\
\hline Tumor number $(<8 / \geq 8)$ & 0.674 & $0.529-0.859$ & 0.001 & 0.656 & $0.509-0.844$ & 0.001 \\
\hline Tumor size $[\mathrm{mm}](<35 / \geq 35)$ & 0.820 & $0.646-1.041$ & 0.102 & 0.863 & $0.661-1.125$ & 0.276 \\
\hline Macrovascular invasion $(-/+)$ & 0.614 & $0.469-0.804$ & $<0.001$ & 0.720 & $0.528-0.982$ & 0.038 \\
\hline Extrahepatic spread $(-/+)$ & 0.842 & $0.661-1.070$ & 0.160 & 0.674 & $0.521-0.872$ & 0.003 \\
\hline Muscle volume (high/low) & 0.745 & $0.586-0.946$ & 0.016 & 0.698 & $0.509-0.959$ & 0.027 \\
\hline
\end{tabular}

ECOG-PS, Eastern Cooperative Oncology Group performance status; HR, hazard ratio; CI, confidence interval. Bold means a $p$-value $<0.05$.

Table 3. Univariate and multivariate analyses for predictors of overall survival $(n=356)$.

\begin{tabular}{|c|c|c|c|c|c|c|}
\hline \multirow{2}{*}{ Factors } & \multicolumn{3}{|c|}{ Univariate Analysis } & \multicolumn{3}{|c|}{ Multivariate Analysis } \\
\hline & HR & $95 \% \mathrm{CI}$ & $p$ Value & HR & $95 \% \mathrm{CI}$ & $p$ Value \\
\hline Age $(<70 / \geq 70)$ & 1.055 & $0.840-1.325$ & 0.643 & 1.002 & $0.792-1.266$ & 0.989 \\
\hline Sex (male/female) & 0.766 & $0.576-1.019$ & 0.067 & 0.661 & $0.491-0.889$ & 0.006 \\
\hline Body mass index $\left[\mathrm{kg} / \mathrm{m}^{2}\right](\geq 22 /<22)$ & 0.918 & $0.728-1.158$ & 0.472 & 1.217 & $0.919-1.612$ & 0.170 \\
\hline ECOG-PS $(-1 / 2-)$ & 0.578 & $0.238-1.404$ & 0.266 & 0.590 & $0.241-1.445$ & 0.248 \\
\hline Child-Pugh class (A/B) & 0.685 & $0.490-0.956$ & 0.026 & 0.701 & $0.496-0.992$ & 0.045 \\
\hline Tumor number $(<8 / \geq 8)$ & 0.625 & $0.496-0.788$ & $<0.001$ & 0.591 & $0.465-0.751$ & $<0.001$ \\
\hline Tumor size $[\mathrm{mm}](<35 / \geq 35)$ & 0.805 & $0.640-1.012$ & 0.063 & 0.863 & $0.670-1.113$ & 0.256 \\
\hline Macrovascular invasion $(-/+)$ & 0.584 & $0.453-0.754$ & $<0.001$ & 0.678 & $0.506-0.907$ & 0.009 \\
\hline Extrahepatic spread $(-/+)$ & 0.771 & $0.612-0.970$ & 0.027 & 0.600 & $0.470-0.766$ & $<0.001$ \\
\hline Muscle volume (high/low) & 0.721 & $0.574-0.907$ & 0.005 & 0.666 & $0.501-0.886$ & 0.005 \\
\hline
\end{tabular}

ECOG-PS, Eastern Cooperative Oncology Group performance status; HR, hazard ratio; CI, confidence interval. Bold means a $p$-value $<0.05$.

\subsection{Prognostic Factor of OS}

On multivariate analysis, the following six factors were found to be independent prognostic factors of OS: sex (HR: 0.717, 95\% CI: 0.524-0.980, $p=0.037$ ), tumor number (HR: 0.615, 95\% CI: 0.476-0.793, $p<0.001$ ), EHS (HR: 0.684, 95\% CI: 0.527-0.887, $p=0.004$ ), 
skeletal muscle volume (HR: 0.545, 95\% CI: 0.393-0.755, $p<0.001$ ), disease control (HR: $0.398,95 \%$ CI: $0.307-0.516, p<0.001$ ), and post-sorafenib therapy (HR: $0.610,95 \%$ CI: $0.472-0.789, p<0.001$ ) (Table 4). Additionally, the results of the post-sorafenib therapies are shown in Figure S3.

Table 4. Univariate and multivariate analyses for prognostic factors of overall survival in patients with progression $(n=320)$.

\begin{tabular}{|c|c|c|c|c|c|c|}
\hline \multirow{2}{*}{ Factors } & \multicolumn{3}{|c|}{ Univariate Analysis } & \multicolumn{3}{|c|}{ Multivariate Analysis } \\
\hline & HR & $95 \% \mathrm{CI}$ & $p$ Value & HR & $95 \% \mathrm{CI}$ & $p$ Value \\
\hline Age $(<70 / \geq 70)$ & 1.092 & $0.860-1.385$ & 0.471 & 0.996 & $0.774-1.282$ & 0.975 \\
\hline Sex (male/female) & 0.718 & $0.531-0.972$ & 0.032 & 0.717 & $0.524-0.980$ & 0.037 \\
\hline Body mass index $\left[\mathrm{kg} / \mathrm{m}^{2}\right](\geq 22 /<22)$ & 0.913 & $0.716-1.164$ & 0.463 & 1.193 & $0.871-1.635$ & 0.271 \\
\hline ECOG-PS $(-1 / 2-)$ & 0.572 & $0.235-1.3908$ & 0.217 & 0.570 & $0.229-1.421$ & 0.228 \\
\hline Child-Pugh class (A / B) & 0.691 & $0.484-0.986$ & 0.041 & 0.685 & $0.469-1.002$ & 0.051 \\
\hline Tumor number $(<8 / \geq 8)$ & 0.673 & $0.528-0.858$ & 0.001 & 0.615 & $0.476-0.793$ & $<0.001$ \\
\hline Tumor size $[\mathrm{mm}](<35 / \geq 35)$ & 0.774 & $0.609-0.984$ & 0.037 & 0.807 & $0.612-1.063$ & 0.127 \\
\hline Macrovascular invasion $(-/+)$ & 0.585 & $0.446-0.766$ & $<0.001$ & 0.858 & $0.621-1.185$ & 0.352 \\
\hline Extrahepatic spread $(-/+)$ & 0.799 & $0.627-1.017$ & 0.068 & 0.684 & $0.527-0.887$ & 0.004 \\
\hline Muscle volume (high/low) & 0.704 & $0.555-0.894$ & 0.004 & 0.545 & $0.393-0.755$ & $<0.001$ \\
\hline Disease control (yes/no) & 0.431 & $0.336-0.552$ & $<0.001$ & 0.398 & $0.307-0.516$ & $<0.001$ \\
\hline Post-sorafenib therapy (yes/no) & 0.575 & $0.449-0.736$ & $<0.001$ & 0.610 & $0.472-0.789$ & $<0.001$ \\
\hline
\end{tabular}

ECOG-PS, Eastern Cooperative Oncology Group performance status; HR, hazard ratio; CI, confidence interval. Bold means a $p$-value $<0.05$.

\section{Discussion}

Several studies on lung, gastric, colorectal, breast, and ovarian cancers have demonstrated that OS, which is considered as the primary outcome in clinical trials on cancer patients who are ineligible for therapy, is strongly correlated with PPS [24-28]. Even in patients with HCC, the same recent studies showed that PPS was strongly correlated with OS. However, PFS was not as strongly correlated with OS as PPS was in HCC patients receiving sorafenib [8] or systemic therapy in randomized controlled trials [29]. This multicenter study also demonstrated a strong correlation between OS and PPS ( $\mathrm{R}=0.908)$ in a large population of HCC patients $(n=273)$ receiving sorafenib, and this correlation was stronger than that between OS and PFS $(R=0.642)$. These results suggest that postprogression treatment after sorafenib failure can also be an important factor for prolonging OS. Currently, there are several MTAs for HCC patients who are resistant to sorafenib, and these drugs reportedly increase survival in these patients $[3,5,6]$. In this study, TACE and HAIC were used as salvage options for post-sorafenib therapy. It has been previously reported that subsequent therapy, including TACE and HAIC, contributes to prolonging PPS after sorafenib therapy [30-32]. We also demonstrate that post-sorafenib therapy is an independent prognostic factor for OS, similar to the PPS prognostic factor (Table S5 and Figure S4). Therefore, these results indicate that sequential therapy after sorafenib failure is important for prolonging PPS.

Previous reports demonstrated that skeletal muscle depletion is a prognostic factor for poor OS in HCC patients receiving sorafenib [17-19]. As skeletal muscle volume is not associated with time to progression [17], which is associated with PFS, it has been suggested that it is associated with PPS. However, there have been few studies on the relationship between skeletal muscle volume and PPS [33]. Although a recent report showed that PPS is significantly associated with pre-sarcopenia at the time of PD in HCC patients receiving sorafenib, the relationship between skeletal muscle volume before sorafenib treatment and PPS has not been established [33]. This is the first study to demonstrate that skeletal muscle volume before sorafenib treatment is a significant predictor of PPS, but not PFS. Additionally, as both skeletal muscle volume and post-sorafenib therapy were found to be independent prognostic factors of PPS and OS, a difference in L-MV and H-MV may also be an important prognostic factor regardless of the presence of post-sorafenib therapy.

The annual rates of skeletal muscle volume decline in cirrhotic patients were reported to be $1.3 \%, 3.5 \%$, and $6.1 \%$ for Child-Pugh classes A, B, and C, respectively [34]. 
In contrast, our previous study found that skeletal muscle volume decreased by $5.8 \%$ 3 months after starting sorafenib treatment, regardless of muscle volume [19]. Another study demonstrated a significant loss of skeletal muscle volume regardless of the presence of disease progression in HCC patients treated with sorafenib or lenvatinib [35]. Thus, compared to treatment with HAIC, treatment with sorafenib may markedly reduce skeletal muscle volume [36] and significantly worsen liver function [37]. Moreover, although skeletal muscle volume is not associated with treatment response to sorafenib and PFS, patients may still require an adequate amount of skeletal muscle volume to receive sorafenib and maintain a Child-Pugh class of A after sorafenib therapy. Therefore, skeletal muscle volume may be considered as an energy reservoir for prolonging PPS in HCC patients receiving sorafenib.

In the era of MTAs, compared to first-line MTA therapy, sequential therapy using MTAs may decrease skeletal muscle volume more significantly; thus, patients with H-MV may have a survival advantage even without achieving disease control with first-line therapy, because skeletal muscle volume and disease control were found to be independent predictors of OS. In addition, it is possible to improve skeletal muscle volume before and during MTA therapy by exercise and interventional nutritional therapy, including branched-chain amino acid and L-carnitine supplementation [38-42]. However, there have been no studies regarding the efficacy of exercise in HCC patients treated with MTAs. Although further investigation is necessary, we highly expect a combination of exercise and nutritional therapy in HCC patients with L-MV to have a synergetic effect.

There are some limitations to this study. First, this is a multicenter, retrospective cohort study. However, it may be impossible to use the same study design in a multicenter prospective study during the same period using multiple MTAs and/or immune checkpoint inhibitors. We consider that this study minimizes the influence of previous systemic therapies and evaluates clinical course following sorafenib therapy. Second, the median value of SMI in males and females was used as the cutoff value in this study (SMI $<45 \mathrm{~cm}^{2} / \mathrm{m}^{2}$ in males and $<38 \mathrm{~cm}^{2} / \mathrm{m}^{2}$ in females). The cutoff values of SMI reportedly vary from 36.2 to $52.4 \mathrm{~cm}^{2} / \mathrm{m}^{2}$ in males and from 29.0 to $39.5 \mathrm{~cm}^{2} / \mathrm{m}^{2}$ in females [43]. The Japan Society of Hepatology (JSH) proposed the criteria for sarcopenia in patients with chronic liver disease [44], wherein skeletal muscle depletion for SMI was defined as a value $<42 \mathrm{~cm}^{2} / \mathrm{m}^{2}$ in males and $<38 \mathrm{~cm}^{2} / \mathrm{m}^{2}$ in females. However, as the optimal cutoff values for CT imaging, which are equivalent to those for bioelectrical impedance analysis $\left(7.0 \mathrm{~kg} / \mathrm{m}^{2}\right.$ in males and $5.7 \mathrm{~kg} / \mathrm{m}^{2}$ in females) based on the Asian Working Group for Sarcopenia criteria [16], were calculated using a receiver-operating characteristic curve analysis of 149 patients with liver cirrhosis or HCC, these cutoff values are provisional. Currently, the cutoff value for SMI in females is the same as the value provided by JSH $\left(38 \mathrm{~cm}^{2} / \mathrm{m}^{2}\right)$, while the cutoff for males is different between this study $\left(45 \mathrm{~cm}^{2} / \mathrm{m}^{2}\right)$ and that given by JSH $\left(42 \mathrm{~cm}^{2} / \mathrm{m}^{2}\right)$. Therefore, our findings suggest that SMI values ranging from 42 to $45 \mathrm{~cm}^{2} / \mathrm{m}^{2}$ in males could constitute a critical range affecting clinical outcomes. Such a range may be important in determining the best cutoff values for skeletal muscle depletion in Japanese patients with HCC. Finally, in this study, post-progression treatment after sorafenib failure mainly used TACE or HAIC, rather than an MTA. The recent trend of post-progression treatment is different from this study, because of the approval of numerous MTAs and ICIs. Patients with progressive loss of skeletal muscle volume after TACE had a significantly poor prognosis $[45,46]$, whereas skeletal muscle depletion was not associated with OS in patients receiving HAIC [36]. Additionally, as opposed to sorafenib, HAIC was associated with a slight decrease in skeletal muscle volume [36]. Conversely, skeletal muscle volume significantly decreased after the indication of sorafenib or lenvatinib [19,35], although there have been no reports on the relationship between skeletal muscle volume and clinical outcomes in patients receiving a combination of atezolizumab and bevacizumab or MTAs other than those mentioned above. Therefore, we believe that an adequate amount of skeletal muscle volume has a positive impact on the PPS regardless of all treatments. 


\section{Conclusions}

This study demonstrates that skeletal muscle volume is a significant predictor of PPS in HCC patients receiving sorafenib. Therefore, upregulation of skeletal muscle volume may improve survival, especially in HCC patients with skeletal muscle depletion.

Supplementary Materials: The following are available online at https://www.mdpi.com/article/ 10.3390/cancers13092247/s1. Figure S1: Enrollment of patients and outcomes, Figure S2: Correlation between overall survival (OS) and either progression-free survival (PFS) or post-progression survival (PPS), Figure S3: Post-sorafenib therapy according to skeletal muscle volume, Figure S4: Post-progression survival according to absence or presence of post-sorafenib therapy, Table S1: Univariate and multivariate analyses of predictors affecting disease control with sorafenib, Table S2: Characteristics of 356 patients with hepatocellular carcinoma according to the skeletal muscle volume, Table S3: Characteristics of 320 patients with hepatocellular carcinoma according to the skeletal muscle volume, Table S4: Univariate and multivariate analyses for predictors of progression-free survival, Table S5: Univariate and multivariate analyses for prognostic factors of post-progression survival.

Author Contributions: Conceptualization, I.S. (Issei Saeki) and T.Y.; Data curation, I.S. (Issei Saeki), Y.Y., T.K. (Tomokazu Kawaoka), S.U., A.H., H.A., K.K., T.K. (Takayuki Kondo), S.O., and T.C.; Formal analysis, I.S. (Issei Saeki), T.Y., Y.Y., R.K., and T.T.; Writing—original draft, I.S. (Issei Saeki), T.Y., and Y.Y.; Writing-review and editing, K.C., N.K., and I.S. (Isao Sakaida). All authors have read and agreed to the published version of the manuscript.

Funding: This research received no external funding.

Institutional Review Board Statement: The study was conducted according to the guidelines of the Declaration of Helsinki and approved by the Institutional Review Board of the Yamaguchi University Hospital (H30-042).

Informed Consent Statement: Patient consent was waived due to the retrospective study design.

Data Availability Statement: The dataset is available from the corresponding author on reasonable request.

Acknowledgments: The authors wish to thank all the collaborators involved in this study.

Conflicts of Interest: H.A. has received honoraria from Eisai and Bayer. S.O. has received grant support, advisory fee, and honoraria from Bayer and Eisai. K.C. has received honoraria from AbbVie, MSD, Gilead Sciences Inc, Bristol Myers Squibb (BMS), Sumitomo Dainippon Pharma, Otsuka, and Tanabe Mitsubishi, and grants and research funding from Sumitomo Dainippon Pharma. N.K. has received grant support, advisory fee, and honoraria from Bayer and Eisai. All other authors have no conflicts of interest to declare.

\section{References}

1. Llovet, J.; Ricci, S.; Mazzaferro, V.; Hilgard, P.; Gane, E.; Blanc, J.; de Oliveira, A.; Santoro, A.; Raoul, J.; Forner, A.; et al. Sorafenib in advanced hepatocellular carcinoma. N. Engl. J. Med. 2008, 359, 378-390. [CrossRef]

2. Finn, R.S.; Qin, S.; Ikeda, M.; Galle, P.R.; Ducreux, M.; Kim, T.Y.; Kudo, M.; Breder, V.; Merle, P.; Kaseb, A.O.; et al. Atezolizumab plus Bevacizumab in Unresectable Hepatocellular Carcinoma. N. Engl. J. Med. 2020, 382, 1894-1905. [CrossRef] [PubMed]

3. Bruix, J.; Qin, S.; Merle, P.; Granito, A.; Huang, Y.H.; Bodoky, G.; Pracht, M.; Yokosuka, O.; Rosmorduc, O.; Breder, V.; et al. Regorafenib for patients with hepatocellular carcinoma who progressed on sorafenib treatment (RESORCE): A randomised, double-blind, placebo-controlled, phase 3 trial. Lancet 2017, 389, 56-66. [CrossRef]

4. Kudo, M.; Finn, R.S.; Qin, S.; Han, K.H.; Ikeda, K.; Piscaglia, F.; Baron, A.; Park, J.W.; Han, G.; Jassem, J.; et al. Lenvatinib versus sorafenib in first-line treatment of patients with unresectable hepatocellular carcinoma: A randomised phase 3 non-inferiority trial. Lancet 2018, 391, 1163-1173. [CrossRef]

5. Zhu, A.X.; Finn, R.S.; Galle, P.R.; Llovet, J.M.; Kudo, M. Ramucirumab in advanced hepatocellular carcinoma in REACH-2: The true value of $\alpha$-fetoprotein. Lancet Oncol. 2019, 20, e191. [CrossRef]

6. Abou-Alfa, G.K.; Meyer, T.; Cheng, A.L.; El-Khoueiry, A.B.; Rimassa, L.; Ryoo, B.Y.; Cicin, I.; Merle, P.; Chen, Y.; Park, J.W.; et al. Cabozantinib in Patients with Advanced and Progressing Hepatocellular Carcinoma. N. Engl. J. Med. 2018, 379, 54-63. [CrossRef]

7. Alsina, A.; Kudo, M.; Vogel, A.; Cheng, A.L.; Tak, W.Y.; Ryoo, B.-Y.; Evans, T.R.J.; López López, C.; Daniele, B.; Misir, S.; et al. Effects of Subsequent Systemic Anticancer Medication Following First-Line Lenvatinib: A Post Hoc Responder Analysis from the Phase 3 REFLECT Study in Unresectable Hepatocellular Carcinoma. Liver Cancer 2020, 9, 93-104. [CrossRef] [PubMed] 
8. Terashima, T.; Yamashita, T.; Takata, N.; Nakagawa, H.; Toyama, T.; Arai, K.; Kitamura, K.; Sakai, Y.; Mizukoshi, E.; Honda, M.; et al. Post-progression survival and progression-free survival in patients with advanced hepatocellular carcinoma treated by sorafenib. Hepatol. Res. 2016, 46, 650-656. [CrossRef] [PubMed]

9. Saeki, I.; Yamasaki, T.; Maeda, M.; Hisanaga, T.; Iwamoto, T.; Fujisawa, K.; Matsumoto, T.; Hidaka, I.; Marumoto, Y.; Ishikawa, T.; et al. Treatment strategies for advanced hepatocellular carcinoma: Sorafenib. World J. Hepatol. 2018, 10, 571-584. [CrossRef]

10. Rosenberg, I.H. Summary comments: Epidemiological and methodological problems in determing nutritional status of older perosons. Am. J. Clin. Nutr. 1989, 50, 3. [CrossRef]

11. Rosenberg, I.H. Sarcopenia: Origins and clinical relevance. J. Nutr. 1997, 127, 990S-991S. [CrossRef] [PubMed]

12. Prado, C.M.; Lieffers, J.R.; McCargar, L.J.; Reiman, T.; Sawyer, M.B.; Martin, L.; Baracos, V.E. Prevalence and clinical implications of sarcopenic obesity in patients with solid tumours of the respiratory and gastrointestinal tracts: A population-based study. Lancet Oncol. 2008, 9, 629-635. [CrossRef]

13. Meza-Junco, J.; Montano-Loza, A.J.; Baracos, V.E.; Prado, C.M.; Bain, V.G.; Beaumont, C.; Esfandiari, N.; Lieffers, J.R.; Sawyer, M.B. Sarcopenia as a prognostic index of nutritional status in concurrent cirrhosis and hepatocellular carcinoma. J. Clin. Gastroenterol. 2013, 47, 861-870. [CrossRef] [PubMed]

14. Fujiwara, N.; Nakagawa, H.; Kudo, Y.; Tateishi, R.; Taguri, M.; Watadani, T.; Nakagomi, R.; Kondo, M.; Nakatsuka, T.; Minami, T.; et al. Sarcopenia, intramuscular fat deposition, and visceral adiposity independently predict the outcomes of hepatocellular carcinoma. J. Hepatol. 2015, 63, 131-140. [CrossRef] [PubMed]

15. Cruz-Jentoft, A.J.; Bahat, G.; Bauer, J.; Boirie, Y.; Bruyère, O.; Cederholm, T.; Cooper, C.; Landi, F.; Rolland, Y.; Sayer, A.A.; et al. Sarcopenia: Revised European consensus on definition and diagnosis. Age Ageing 2019, 48, 16-31. [CrossRef]

16. Chen, L.K.; Woo, J.; Assantachai, P.; Auyeung, T.W.; Chou, M.Y.; Iijima, K.; Jang, H.C.; Kang, L.; Kim, M.; Kim, S.; et al. Asian Working Group for Sarcopenia: 2019 Consensus Update on Sarcopenia Diagnosis and Treatment. J. Am. Med. Dir. Assoc. 2020, 21, 300-307.e302. [CrossRef]

17. Hiraoka, A.; Hirooka, M.; Koizumi, Y.; Izumoto, H.; Ueki, H.; Kaneto, M.; Kitahata, S.; Aibiki, T.; Tomida, H.; Miyamoto, Y.; et al. Muscle volume loss as a prognostic marker in hepatocellular carcinoma patients treated with sorafenib. Hepatol. Res. 2017, 47, 558-565. [CrossRef] [PubMed]

18. Imai, K.; Takai, K.; Hanai, T.; Ideta, T.; Miyazaki, T.; Kochi, T.; Suetsugu, A.; Shiraki, M.; Shimizu, M. Skeletal muscle depletion predicts the prognosis of patients with hepatocellular carcinoma treated with sorafenib. Int. J. Mol. Sci. 2015, 16, 9612-9624. [CrossRef] [PubMed]

19. Saeki, I.; Yamasaki, T.; Maeda, M.; Kawano, R.; Hisanaga, T.; Iwamoto, T.; Matsumoto, T.; Hidaka, I.; Ishikawa, T.; Takami, T.; et al. No Muscle Depletion with High Visceral Fat as a Novel Beneficial Biomarker of Sorafenib for Hepatocellular Carcinoma. Liver Cancer 2018, 7, 359-371. [CrossRef]

20. Forner, A.; Reig, M.; Bruix, J. Hepatocellular carcinoma. Lancet 2018, 391, 1301-1314. [CrossRef]

21. Mitsiopoulos, N.; Baumgartner, R.N.; Heymsfield, S.B.; Lyons, W.; Gallagher, D.; Ross, R. Cadaver validation of skeletal muscle measurement by magnetic resonance imaging and computerized tomography. J. Appl. Physiol. (1985) 1998, 85, 115-122. [CrossRef] [PubMed]

22. Eisenhauer, E.A.; Therasse, P.; Bogaerts, J.; Schwartz, L.H.; Sargent, D.; Ford, R.; Dancey, J.; Arbuck, S.; Gwyther, S.; Mooney, M.; et al. New response evaluation criteria in solid tumours: Revised RECIST guideline (version 1.1). Eur. J. Cancer 2009, 45, $228-247$. [CrossRef] [PubMed]

23. Tokunaga, K.; Matsuzawa, Y.; Kotani, K.; Keno, Y.; Kobatake, T.; Fujioka, S.; Tarui, S. Ideal body weight estimated from the body mass index with the lowest morbidity. Int. J. Obes. 1991, 15, 1-5.

24. Hayashi, H.; Okamoto, I.; Morita, S.; Taguri, M.; Nakagawa, K. Postprogression survival for first-line chemotherapy of patients with advanced non-small-cell lung cancer. Ann. Oncol. 2012, 23, 1537-1541. [CrossRef] [PubMed]

25. Kawakami, H.; Okamoto, I.; Hayashi, H.; Taguri, M.; Morita, S.; Nakagawa, K. Postprogression survival for first-line chemotherapy in patients with advanced gastric cancer. Eur. J. Cancer 2013, 49, 3003-3009. [CrossRef]

26. Petrelli, F.; Barni, S. Correlation of progression-free and post-progression survival with overall survival in advanced colorectal cancer. Ann. Oncol. 2013, 24, 186-192. [CrossRef]

27. Saad, E.D.; Katz, A.; Buyse, M. Overall survival and post-progression survival in advanced breast cancer: A review of recent randomized clinical trials. J. Clin. Oncol. 2010, 28, 1958-1962. [CrossRef] [PubMed]

28. Shimokawa, M.; Kogawa, T.; Shimada, T.; Saito, T.; Kumagai, H.; Ohki, M.; Kaku, T. Overall survival and post-progression survival are potent endpoint in phase III trials of second/third-line chemotherapy for advanced or recurrent epithelial ovarian cancer. J. Cancer 2018, 9, 872-879. [CrossRef]

29. Terashima, T.; Yamashita, T.; Toyama, T.; Arai, K.; Kawaguchi, K.; Kitamura, K.; Sakai, Y.; Mizukoshi, E.; Honda, M.; Kaneko, S. Surrogacy of Time to Progression for Overall Survival in Advanced Hepatocellular Carcinoma Treated with Systemic Therapy: A Systematic Review and Meta-Analysis of Randomized Controlled Trials. Liver Cancer 2019, 8, 130-139. [CrossRef] [PubMed]

30. Terashima, T.; Yamashita, T.; Horii, R.; Arai, K.; Kawaguchi, K.; Kitamura, K.; Sakai, Y.; Mizukoshi, E.; Honda, M.; Kaneko, S. Potential efficacy of therapies targeting intrahepatic lesions after sorafenib treatment of patients with hepatocellular carcinoma. BMC Cancer 2016, 16, 338. [CrossRef] 
31. Kondo, M.; Numata, K.; Hara, K.; Nozaki, A.; Fukuda, H.; Chuma, M.; Maeda, S.; Tanaka, K. Treatment of Advanced Hepatocellular Carcinoma after Failure of Sorafenib Treatment: Subsequent or Additional Treatment Interventions Contribute to Prolonged Survival Postprogression. Gastroenterol. Res. Pract. 2017, 2017, 5728946. [CrossRef]

32. Terashima, T.; Yamashita, T.; Arai, K.; Sunagozaka, H.; Kitahara, M.; Nakagawa, H.; Kagaya, T.; Mizukoshi, E.; Honda, M.; Kaneko, S. Feasibility and efficacy of hepatic arterial infusion chemotherapy for advanced hepatocellular carcinoma after sorafenib. Hepatol. Res. 2014, 44, 1179-1185. [CrossRef] [PubMed]

33. Cheng, T.Y.; Lee, P.C.; Chen, Y.T.; Chao, Y.; Hou, M.C.; Huang, Y.H. Pre-sarcopenia determines post-progression outcomes in advanced hepatocellular carcinoma after sorafenib failure. Sci. Rep. 2020, 10, 18375. [CrossRef] [PubMed]

34. Hanai, T.; Shiraki, M.; Ohnishi, S.; Miyazaki, T.; Ideta, T.; Kochi, T.; Imai, K.; Suetsugu, A.; Takai, K.; Moriwaki, H.; et al. Rapid skeletal muscle wasting predicts worse survival in patients with liver cirrhosis. Hepatol. Res. 2016, 46, 743-751. [CrossRef] [PubMed]

35. Uchikawa, S.; Kawaoka, T.; Namba, M.; Kodama, K.; Ohya, K.; Morio, K.; Nakahara, T.; Murakami, E.; Tsuge, M.; Hiramatsu, A.; et al. Skeletal Muscle Loss during Tyrosine Kinase Inhibitor Treatment for Advanced Hepatocellular Carcinoma Patients. Liver Cancer 2020, 9, 148-155. [CrossRef]

36. Saeki, I.; Yamasaki, T.; Maeda, M.; Hisanaga, T.; Iwamoto, T.; Matsumoto, T.; Hidaka, I.; Ishikawa, T.; Takami, T.; Sakaida, I. Effect of body composition on survival benefit of hepatic arterial infusion chemotherapy for advanced hepatocellular carcinoma: A comparison with sorafenib therapy. PLoS ONE 2019, 14, e0218136. [CrossRef] [PubMed]

37. Terashima, T.; Yamashita, T.; Arai, K.; Kawaguchi, K.; Kitamura, K.; Sakai, Y.; Mizukoshi, E.; Honda, M.; Kaneko, S. Beneficial Effect of Maintaining Hepatic Reserve during Chemotherapy on the Outcomes of Patients with Hepatocellular Carcinoma. Liver Cancer 2017, 6, 236-249. [CrossRef]

38. Takami, T.; Yamasaki, T.; Saeki, I.; Matsumoto, T.; Suehiro, Y.; Sakaida, I. Supportive therapies for prevention of hepatocellular carcinoma recurrence and preservation of liver function. World J. Gastroenterol. 2016, 22, 7252-7263. [CrossRef]

39. Hashida, R.; Kawaguchi, T.; Koya, S.; Hirota, K.; Goshima, N.; Yoshiyama, T.; Otsuka, T.; Bekki, M.; Iwanaga, S.; Nakano, D.; et al. Impact of cancer rehabilitation on the prognosis of patients with hepatocellular carcinoma. Oncol. Lett. 2020, 19, $2355-2367$. [CrossRef]

40. Ohara, M.; Ogawa, K.; Suda, G.; Kimura, M.; Maehara, O.; Shimazaki, T.; Suzuki, K.; Nakamura, A.; Umemura, M.; Izumi, T.; et al. L-Carnitine Suppresses Loss of Skeletal Muscle Mass in Patients With Liver Cirrhosis. Hepatol. Commun. 2018, 2, 906-918. [CrossRef]

41. Takeda, H.; Nishikawa, H.; Iguchi, E.; Ohara, Y.; Sakamoto, A.; Saito, S.; Nishijima, N.; Nasu, A.; Komekado, H.; Kita, R.; et al. Effect of treatment with branched-chain amino acids during sorafenib therapy for unresectable hepatocellular carcinoma. Hepatol. Res. 2014, 44, 302-312. [CrossRef] [PubMed]

42. Imanaka, K.; Ohkawa, K.; Tatsumi, T.; Katayama, K.; Inoue, A.; Imai, Y.; Oshita, M.; Iio, S.; Mita, E.; Fukui, H.; et al. Impact of branched-chain amino acid supplementation on survival in patients with advanced hepatocellular carcinoma treated with sorafenib: A multicenter retrospective cohort study. Hepatol. Res. 2016, 46, 1002-1010. [CrossRef] [PubMed]

43. Chang, K.V.; Chen, J.D.; Wu, W.T.; Huang, K.C.; Hsu, C.T.; Han, D.S. Association between Loss of Skeletal Muscle Mass and Mortality and Tumor Recurrence in Hepatocellular Carcinoma: A Systematic Review and Meta-Analysis. Liver Cancer 2018, 7, 90-103. [CrossRef]

44. Nishikawa, H.; Shiraki, M.; Hiramatsu, A.; Moriya, K.; Hino, K.; Nishiguchi, S. Japan Society of Hepatology guidelines for sarcopenia in liver disease (1st edition): Recommendation from the working group for creation of sarcopenia assessment criteria. Hepatol. Res. 2016, 46, 951-963. [CrossRef]

45. Kobayashi, T.; Kawai, H.; Nakano, O.; Abe, S.; Kamimura, H.; Sakamaki, A.; Kamimura, K.; Tsuchiya, A.; Takamura, M.; Yamagiwa, S.; et al. Rapidly declining skeletal muscle mass predicts poor prognosis of hepatocellular carcinoma treated with transcatheter intra-arterial therapies. BMC Cancer 2018, 18, 756. [CrossRef]

46. Fujita, M.; Takahashi, A.; Hayashi, M.; Okai, K.; Abe, K.; Ohira, H. Skeletal muscle volume loss during transarterial chemoembolization predicts poor prognosis in patients with hepatocellular carcinoma. Hepatol. Res. 2019, 49, 778-786. [CrossRef] [PubMed] 